\title{
MicroRNA-22 functions as a tumor suppressor by targeting SIRT1 in renal cell carcinoma
}

\author{
SHOULIN ZHANG ${ }^{1}$, DONGMEI ZHANG ${ }^{2},{\text { CHUNGUANG } \text { YI }^{3} \text {, YINPING WANG }}^{1}$, \\ HONGAN WANG ${ }^{1}$ and JIAN WANG ${ }^{1}$
}

\begin{abstract}
${ }^{1}$ Internal Medicine Department, The Affiliated Hospital to Changchun University of Chinese Medicine, Changchun, Jilin 130021; ${ }^{2}$ Scientific Research Office, The Affiliated Hospital to Changchun University of Chinese Medicine, Changchun, Jilin 130021; ${ }^{3}$ College of Pharmacy, Changchun University of Chinese Medicine, Changchun Jingyue National

High-Tech Industrial Development Zone, Changchun, Jilin 130117, P.R. China
\end{abstract}

Received June 19, 2015; Accepted July 30, 2015

DOI: $10.3892 /$ or.2015.4333

\begin{abstract}
Accumulating evidence demonstrates that microRNA-22 (miR-22) was deregulated in many types of cancers and was involved in various cellular processes related to carcinogenesis. However, the exact roles and mechanisms of miR-22 remain unknown in human renal cell carcinoma (RCC). Here, the relationship between miR-22 expression pattern and clinicopathological features of patients with EOC were determined by real-time quantitative RT-PCR (qRT-PCR). Furthermore, the role of miR-22 and possible molecular mechanisms in EOC were investigated by several in vitro approaches and in a nude mouse model. Results from qRT-PCR showed that miR-22 was significantly downregulated in RCC samples compared with corresponding non-cancerous tissues, which was significantly associated with tumor stage and lymph node metastasis. Functional study demonstrated that enforced overexpression of miR-22 in renal cancer cells inhibited proliferation, migration and invasion, and induced cell apoptosis in vitro, and suppressed tumor growth in vivo. In addition, SIRT1 was identified as a direct target of miR-22 by a luciferase reporter assay. Overexpression of miR-22 activated p53 and its downstream target p21 and PUMA, and the apoptosis markers cleaved CASP3 and PARP, and inhibited epithelial-mesenchymal transition (EMT). These findings showed that miR-22 functioned as tumor suppressor in RCC and blocked RCC growth and metastasis by directly targeting SIRT1 in RCC, indicating a potential novel therapeutic role in RCC treatment.
\end{abstract}

Correspondence to: Dr Jian Wang, Internal Medicine Department, The Affiliated Hospital to Changchun University of Chinese Medicine, 1478 Gongnong Road, Changchun, Jilin 130021, P.R. China

E-mail: wangjian542b@sina.com

Key words: renal cell carcinoma, miR-22, SIRT1, p53

\section{Introduction}

Renal cell carcinoma ( $\mathrm{RCC}$ ) is the most lethal urologic tumor, with estimated 63,920 new cases and 13,860 deaths in 2014 (1). Clear cell renal cell carcinoma (ccRCC), originating from the renal proximal tubule, is the most common subtype of RCC and accounts for $\sim 75-80 \%$ of these tumors with the highest rates of local invasion, metastasis, mortality and refractory to current treatments $(2,3)$. Despite increased early detection of RCC and more frequent surgery, $30 \%$ of patients develop metastases after surgery (3-5). Therefore, it is urgent to understand the underlying molecular mechanisms involved in the pathogenesis of RCC for developing cancer prevention strategies and possible guiding disease management in the clinic.

MicroRNAs (miRNAs) are a class of small, single stranded, non-coding RNA molecules of 19-24 nucleotides that bind to complementary sequences in the $3^{\prime}$ untranslated regions (UTR) of their target mRNAs and induce mRNA degradation or translational repression $(6,7)$. miRNAs have been implicated in the regulation of various biological processes, such as cell proliferation, migration, invasion, apoptosis, metabolism, and cellular differentiation $(8,9)$. Furthermore, miRNAs have recently been reported to function as both oncogenes and suppressors of tumor progression, and to play crucial roles in human tumorigenesis and/or metastasis by directly targeting oncogenes or tumor suppressor genes (10). Therefore, miRNA expression can contribute to the diagnosis and classification of cancers, and contribute to develop novel avenues for targeted therapy (11).

miR-22, a 22-nt non-coding RNA, has been found to be frequently downregulated in several cancers, such as breast cancer (12), osteosarcoma (13), esophageal squamous cell carcinoma (14), gastric cancer (15) and colorectal cancer (16). Accumulating evidence shows that miR-22 plays key roles in the regulation of cell growth, migration, chemosensitivity and epithelial-mesenchymal transition in cancers (17). For RCC, recently it was reported that miR-22 was significantly downregulated in RCC tumor tissues as compared with adjacent normal tissues (18). However, our knowledge of the clinicopathological impact and the exact roles of the miR-22 
in EOC and the underlying molecular mechanisms remains unclear.

The aim of this study was to investigate the involvement of miR-22 in renal cancer. We found that the expression level of miR-22 decreased in ccRCC tissues and was inversely associated with histologic grade, tumor stage, lymph node metastasis, and distant metastasis. Function studies demonstrated that miR-22 acts as a tumor suppressor by affecting renal cancer cell proliferation, apoptosis, migration and invasion. Furthermore, SIRT1 was identified as a direct functional target of miR-22. Overexpression of miR-22 activated p53 and its downstream target p21 and PUMA, as well as the apoptosis markers cleaved CASP3 and PARP, and inhibited epithelialmesenchymal transition (EMT). These findings indicated that miR-22 has a potential role in the therapy of RCC.

\section{Materials and methods}

Human RCC clinical specimens. A total of 50 primary RCC tissues and matched adjacent normal tissues were obtained from patients who underwent radical nephrectomy in the TCM Nephropathy, the Affiliated Hospital, Changchun University of Chinese Medicine between July 2012 to December 2014. None of the patients had received chemotherapy or radiotherapy prior to surgery. After surgical resection, tumor tissues and adjacent normal tissues were collected and stored at $-80^{\circ} \mathrm{C}$ until use. Use of clinical sample cohorts in this study was approved by the Institution Research Ethics Committee of Changchun University of Chinese Medicine. All patients gave written consent for their information to be stored in the hospital database and used for this study. The clinical and pathological information from patient records was gathered, and is listed in Table I.

Cell culture. Immortalized normal human proximal tubule epithelial cell line HK-2 was purchased from the American Type Culture Collection (ATCC; USA). Four human RCC cell lines 786-O, ACHN, Caki-1, and Caki-2 were obtained from the Cell Bank of Type Culture Collection of Chinese Academy of Sciences (Shanghai, China), All cells were cultured in RPMI-1640 medium with 10\% fetal bovine serum (FBS) (both from Gibco), $50 \mathrm{U} / \mathrm{ml}$ penicillin or $50 \mu \mathrm{g} / \mathrm{ml}$ streptomycin at $37^{\circ} \mathrm{C}$ in a $5 \% \mathrm{CO}_{2}$ humidified incubator.

Quantitative reverse transcription-polymerase chain reaction $(q R T-P C R)$. Total RNA was extracted from tissue and cultured cells using TRIzol (Invitrogen, USA) according to the manufacturer's instructions. For mRNA reverse transcription, cDNA was synthesized using the PrimeScript RT reagent kit (Takara Bio, Japan). Quantitative real-time polymerase chain reaction (RT-qPCR) analysis of the SIRT1 expression in mRNA level was performed using Fast SYBR Green Master Mix (Applied Biosystems, Foster City, CA, USA) under ABI 7900 Sequence Detection system (Life Technologies, NY, USA). GAPDH were used as endogenous controls for miRNA GAPDH. The specific primers for GAPDH and SIRT1 are as follows: GAPDH (sense), 5'-TCAACGACCACTTTGTCAAGCTCA-3' and antisense, 5'-GCTGGTGGTCCAGGGGTCTTACT-3'; and SIRT1 (sense), 5'-GCCAGAGTCCAAGTTTAGAAGA-3' and antisense, 5'-CCATCAGTCCCAAATCCAG-3'.
Table I. Correlation between clinicopathological features and miR-22 expression in $50 \mathrm{RCC}$ tissues.

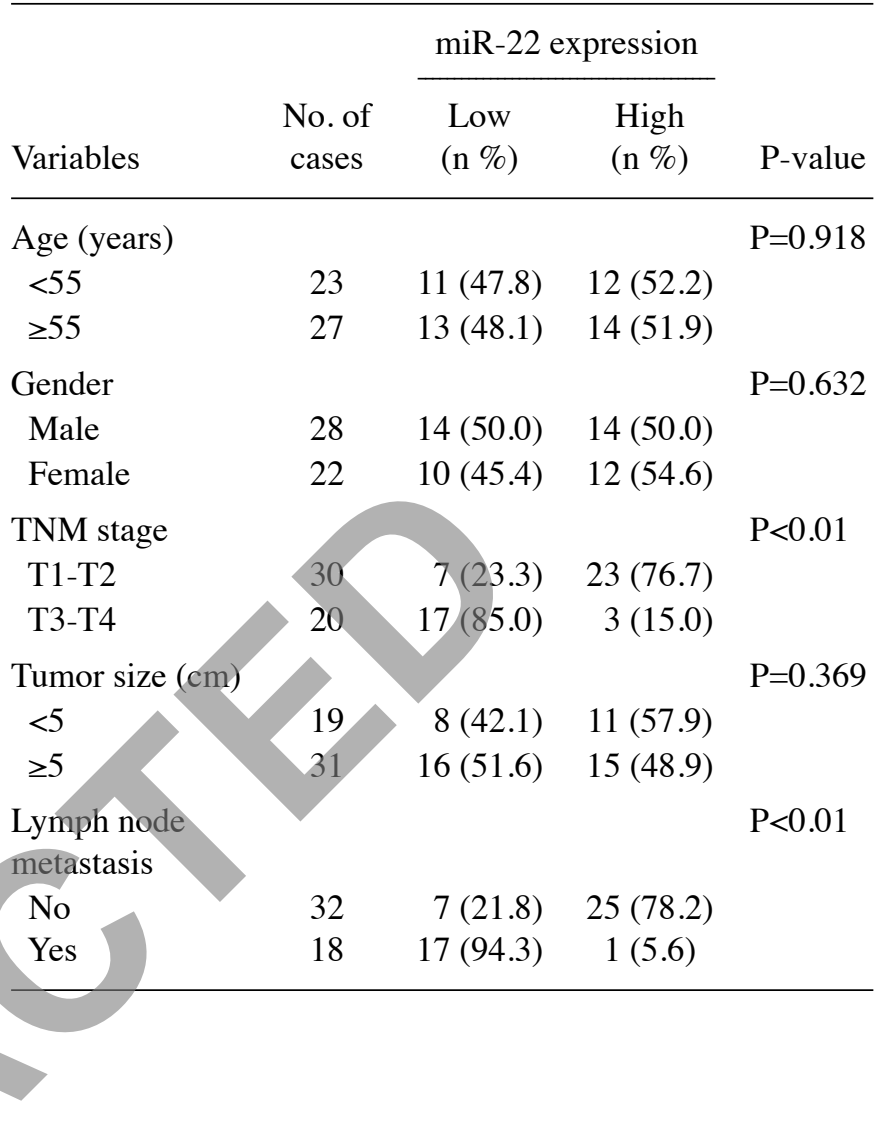

Total miRNA extraction was performed from tissue and cells using the mirVana miRNA Isolation kit (Ambion, Austin, TX, USA). For miRNA reverse transcription, cDNA was synthesized using One Step PrimeScript miRNA cDNA Synthesis kit (Qiagen, Valencia, CA, USA) according to the manufacturer's instructions. Quantification of miR-22 expression was performed using the mirVana qRT-PCR miRNA Detection kit (Ambion). U6 were used as endogenous controls for miRNA. The comparative $2^{-\Delta \Delta C t}$ method was used for relative quantification and statistical analysis. All experiments were performed in triplicate.

Transient transfection of miRNA mimics. The miRNA-22 mimic and corresponding negative control mimics (miRNC) were purchased from GenePharma (Shanghai, China). Transfection of miR-22 mimic or miR-NC at the final concentration of $100 \mathrm{nM}$ into RCC cells was performed using Lipofectamine 2000 (Invitrogen) according to the manufacturer's procedure. Transfection efficiency was evaluated in each experiment by qRT-PCR $48 \mathrm{~h}$ after transfection.

Cell proliferation and colony formation assay. To assess cell proliferation, $5 \times 10^{3} 786-\mathrm{O}$ cells were plated in 96 -well plates overnight and transfected with the miR-22 mimic or miR-NC. Proliferation was determined by 3-(4,5-dimethylthiazol-2-yl)-2-5 diphenyltetrazolium bromide (MTT; Sigma, USA) assay at 24, 48, 72, and $96 \mathrm{~h}$ after transfection, and the absorbance of samples was measured with a microplate reader (Bio-Rad, Gaithersburg, MD, USA) at $490 \mathrm{~nm}$. All experiments were performed in three replicates and were repeated three times independently. 
For colony formation assay, 786-O cells were transfected with miR-22 mimic or miR-NC in $60-\mathrm{mm}$ culture dishes. After $24 \mathrm{~h}, 1 \times 10^{3}$ cells were transferred to 6 -well plates and incubated for 2 weeks. Then, cells were rinsed with PBS and fixed with $1 \%$ formaldehyde for $30 \mathrm{~min}$ at room temperature. Fixed cells were stained with $1 \%$ crystal violet. Finally the clones were photographed and counted. The percentage of the colony formation was calculated by adjusting control (miR-NC) to $100 \%$.

Cell cycle assay. To assess cell cycle distribution and cell apoptosis, $2 \times 10^{4} 786-\mathrm{O}$ cells were plated in 6-well plates and transfected with miR-22 or miR-NC. After transfection, the cells were collected by trypsinization, fixed in $70 \%$ ice-cold ethanol overnight, then washed with PBS and stained with propidium iodide (PI, $50 \mathrm{mg} / \mathrm{ml}$; Sigma) in PBS supplemented with RNase $(50 \mathrm{mg} / \mathrm{ml})$ for $30 \mathrm{~min}$ in the dark at room temperature (RT). Analyses of cell cycle distribution were performed by flow cytometer following the manufacturer's guidelines (BD Biosciences).

Apoptosis assay. Cell apoptosis analysis was performed with Annexin V-FITC Apoptosis Detection kit I (BD Biosciences). In briefly, $2 \times 10^{4} 786-\mathrm{O}$ cells transfected with miR-22 mimic or miR-NC were suspended in RPMI-1640 medium. The cells were re-suspended in $500 \mu 1$ cold binding buffer with $2 \mu 1$ Annexin V-FITC, and incubated for $15 \mathrm{~min}$ in the dark at room temperature. Cells were re-suspended in $500 \mu \mathrm{l}$ cold binding buffer with $10 \mu \mathrm{l} \mathrm{PI}$, incubated for $4 \mathrm{~h}$ and analyzed by flow cytometry (BD Biosciences).

Scratch migration assay. To assess the effect of miR-22 on cell migration, 786-O cells transfected with miR-22 mimic or miR-NC were seeded into 6-well plates and cultured overnight. Before scratching, cells were starved in medium with $1 \%$ FBS for $24 \mathrm{~h}$. Thereafter, similar sized wounds were scratched into the monolayer using a sterile plastic micropipette tip. Wounded monolayer cells were washed three times by PBS to remove cell debris and then cultured. Migration of cells into the wound was observed at 0 and $24 \mathrm{~h}$ using an IX51 inverted microscope (Olympus, Tokyo, Japan). Individual cells were quantified as an average of at least five fields for each experiment. Each experiment was performed three times independently.

Invasion assay. For the Transwell invasion assay, $3 \times 10^{4}$ transfected cells suspended in serum-free medium were added to the upper chamber. In the bottom chamber, medium containing $10 \%$ FBS was added as a chemoattractant. After 24-h culture at $37^{\circ} \mathrm{C}$, the cells remaining in the upper chamber or on the upper membrane was removed with a sterile swab. Invaded cells of the bottom chamber were fixed with $70 \%$ ethanol for $30 \mathrm{~min}$ and stained with $0.2 \%$ crystal violet (Sigma) for $10 \mathrm{~min}$. Invading cells were counted by taking photomicrographs in five random fields.

Vector construction and luciferase reporter assay. A human SIRT1 3'UTR was amplified from renal cancer cell line cDNAs and cloned into the XhoI/NotI site of a psiCHECK-2 vector (Promega, Madison, WI, USA). For mutagenesis of the
miR-22-binding site, a QuikChange Site-Directed Mutagenesis kit (Agilent Technologies, Palo Alto, CA, USA) was used following the manufacturer's instructions.

For luciferase activity assay, 786-O cells were seeded into 12-well plates overnight before transfection, and then co-transfected with $200 \mathrm{ng}$ of psiCHECK-2 vectors, which harbored SIRT1 3'UTR wild-type or mutant constructs, and $200 \mathrm{nM}$ of miR-22 or miR-NC. Forty-eight hours after transfection, luciferase activity was measured with a Dual-Luciferase assay kit (Promega) according to the manufacturer's instructions. Renilla luciferase activity was normalized to firefly luciferase activity.

Western blot analysis. Tissue sample and cells were collected and homogenized with RIPA lysis buffer $(150 \mathrm{mM} \mathrm{NaCl}, 0.5 \%$ sodium deoxycholate, $0.1 \% \mathrm{SDS}, 1 \%$ Igepal, $50 \mathrm{mM}$ Tris- $\mathrm{HCl}$ pH 8.0, 2 mM EDTA) supplemented with $1 \mathrm{mM}$ DTT, $1 \mathrm{mM}$ Pefabloc, $1 \mathrm{mM} \mathrm{NaV}_{3}, 10 \mathrm{mM} \mathrm{NaF}$ and $1 \mathrm{X}$ complete mini protease inhibitor cocktail tablets. Total protein concentration was detected using a bicinchoninic acid (BCA) protein assay kit (Boster, China). Thirty micrograms of protein/lane were resolyed on $8-15 \%$ sodium dodecylsulfate-polyacrylamide gels (SDS-PAGE), and then transferred onto the nitrocellulose membrane (Bio-Rad, Munich, Germany). The membrane was incubated with 5\% non-fat skim milk for $2 \mathrm{~h}$ at room temperature. The membrane was incubated with the following antibodies overnight at $4^{\circ} \mathrm{C}$ : SIRT1, E-cadherin, $\mathrm{N}$-cadherin, vimentin, p21, p53, Ac-p53(382), PUMA, PARP, GAPDH and cleaved CASP3. GAPDH was used as the internal control. All antibodies were from Santa Cruz Biotechnology (Santa Cruz, CA, USA). Then the membrane was incubated with the corresponding horseradish peroxidase (HRP)-conjugated secondary antibody (Santa Cruz Biotechnology) for $2 \mathrm{~h}$ at room temperature. Proteins were visualized with ECL chemiluminescent kit (ECL Plus; Thermo Scientific).

In vivo nude mouse tumorigenesis assay. Male BALB/c nude mice (five weeks old, 18-20 g) were obtained from Experiments Animal Center of Changchun Biological Institute (Changchun, China), and maintained under specific pathogen-free conditions. This study and all experimental protocols were approved by the Animal Ethics Committee of Changchun University of Chinese Medicine (Changchun, China).

About $2 \times 10^{6}$ logarithmically growing $786-\mathrm{O}$ cells stably expressing miR-22 or miR-NC, were suspended in $100 \mu \mathrm{l}$ of medium (containing 10\% Matrigel) then injected into the flanks of mice $(n=10)$, respectively. Tumor volume was measured every five days by digital Vernier calipers, and was calculated according to the following formula: $[\pi / 6 \mathrm{x}$ length $\mathrm{x}$ width $\mathrm{x}$ height]. Thirty days after inoculation, mice were sacrificed, and tumors were removed and weighed. Part of the tumor tissues was harvested for analysis of the expression of SIRT1 by western blot analysis.

Statistical analysis. All the data are shown as mean \pm SD (standard deviation) from at least three independent experiments. The difference was determined by two-tailed Student's t-test. Statistical analysis was performed with GraphPad Prism 5.0 (GraphPad Software, San Diego, CA, USA). In all cases, $\mathrm{P}<0.05$ was considered statistically significant. 
A

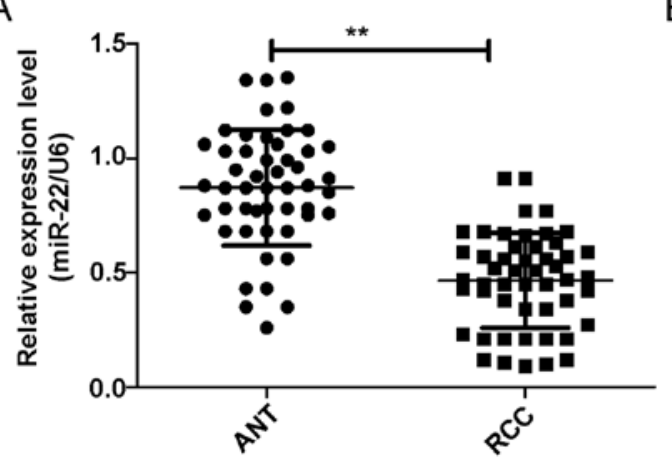

B

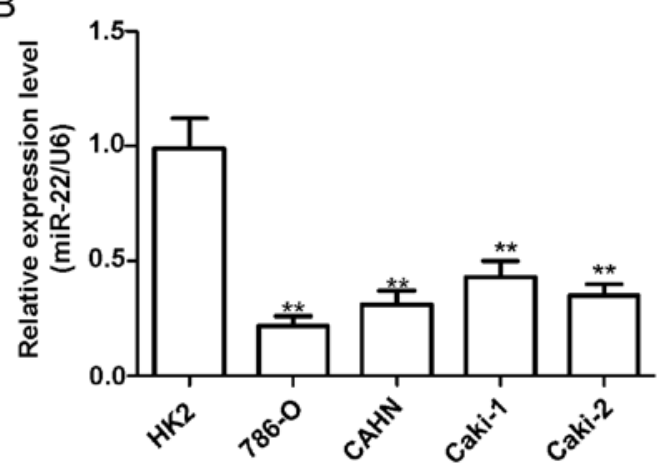

Figure 1. miR-22 expression was downregulated in human renal cancer tissue and cell lines. (A) Expression of miR-22 in 4 renal cancer cell lines and human proximal tubule epithelial cell line HK-2 were determined by qRT-PCR. ${ }^{* *} \mathrm{P}<0.01$ vs. HK-2. (B) Expression of miR-22 in RCC tissues and adjacent normal tissues (ANT) were determined by qRT-PCR. ${ }^{*} \mathrm{P}<0.05,{ }^{* *} \mathrm{P}<0.01$ vs. ANT.

A

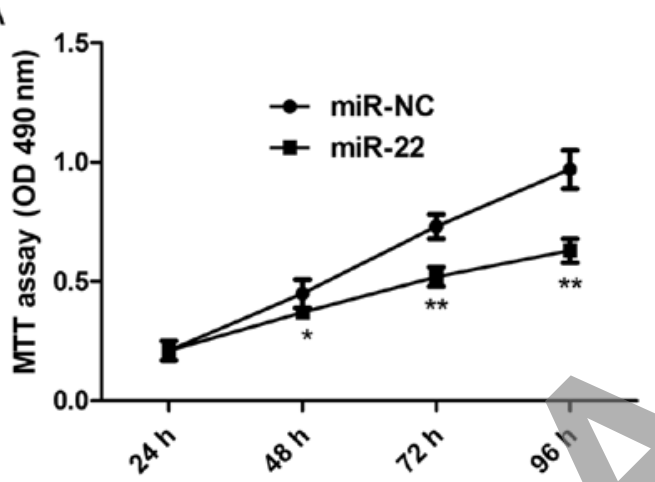

C
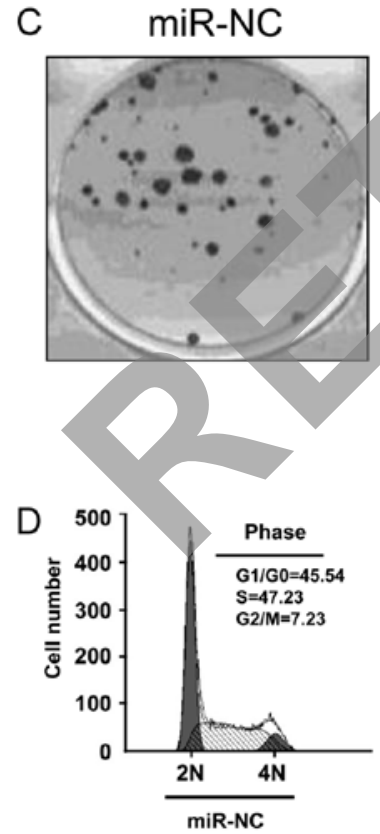
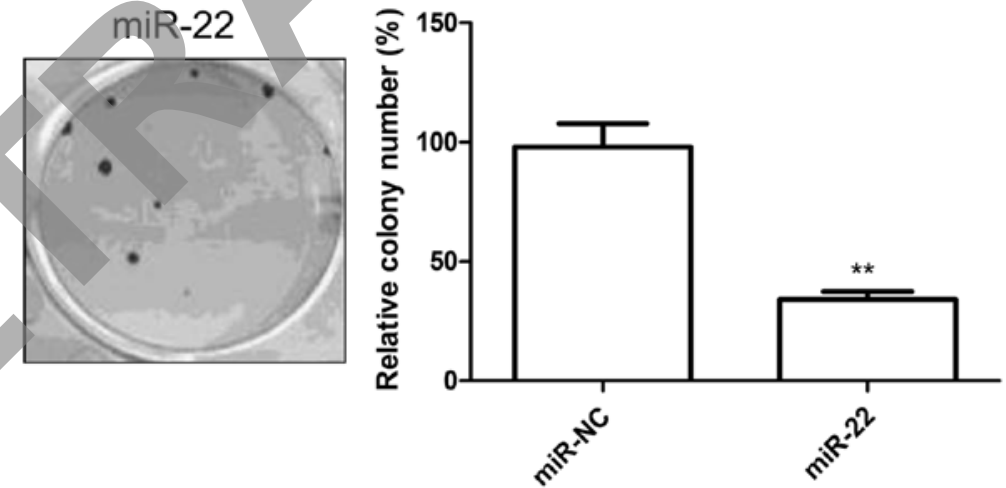

E
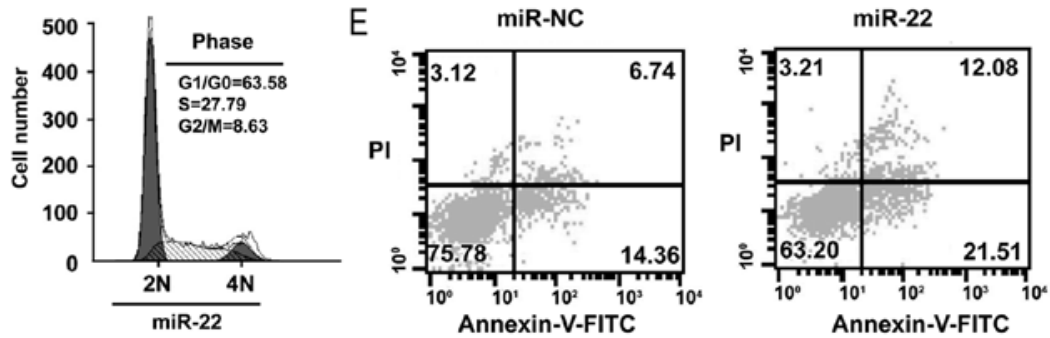

$\mathrm{B}$

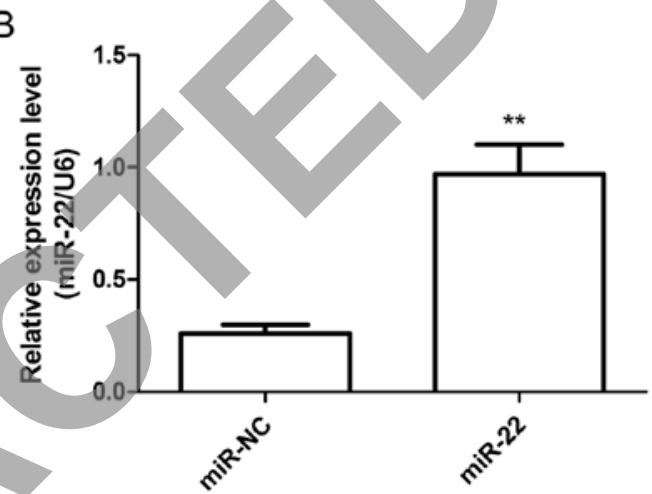

Figure 2. Overexpression of miR-22 inhibits cells proliferation and colony formation, induces cell arrest at G0/G1 phase and cell apoptosis in RCC cells (A) miR-22 mimic restored miR-22 expression in 786-O cells. (B) Cell proliferation was determined in 786-O cells transfected with miR-22 mimic or miR-NC by MTT assay. (C) Cell colony formation was determined in 786-O cells transfected with miR-22 mimic or miR-NC. (D) Cell cycle distribution was determined in 786-O cells transfected with miR-22 mimic or miR-NC by FACS analysis. (E) Cell apoptosis was determined in 786-O cells transfected with miR-22 mimic or miR-NC by FACS analysis. ${ }^{*} \mathrm{P}<0.05,{ }^{* *} \mathrm{P}<0.01$ vs. miR-NC.

\section{Results}

miR-22 expression is downregulated in renal cancer tissue and cell lines. To investigate the expression levels of miR-22 in renal cancer, the expression level of miR-22 in four RCC cell lines was first analyzed by qRT-PCR. In comparison to immortalized normal proximal tubule epithelial cell line HK-2, miR-22 expression was downregulated in all four renal cancer 
A
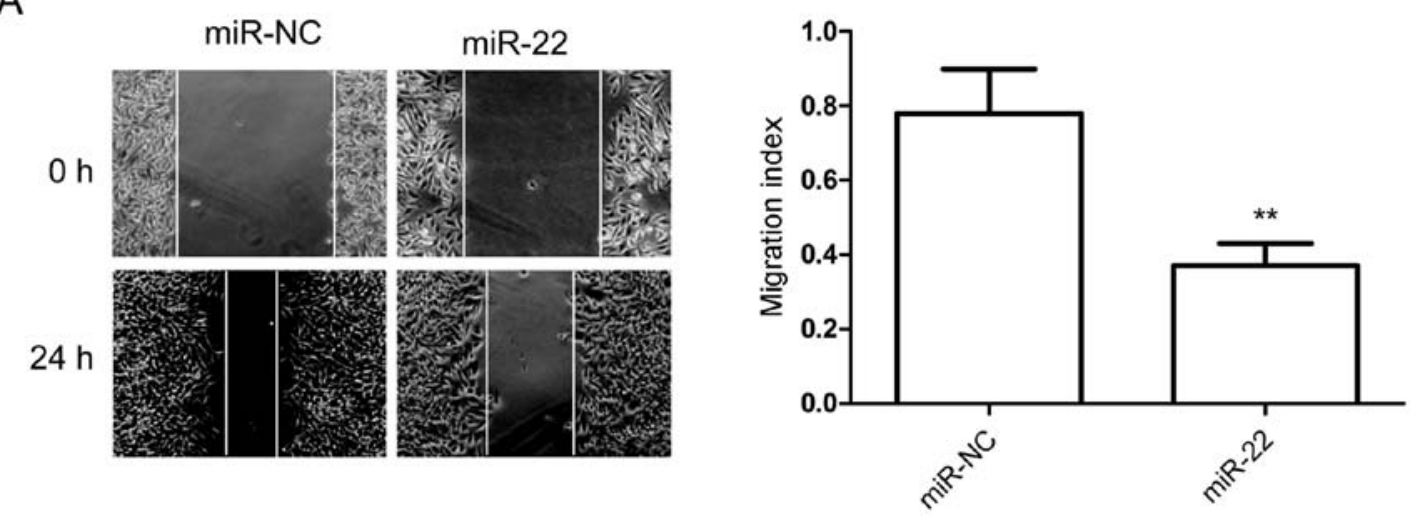

B

miR-NC

miR-22
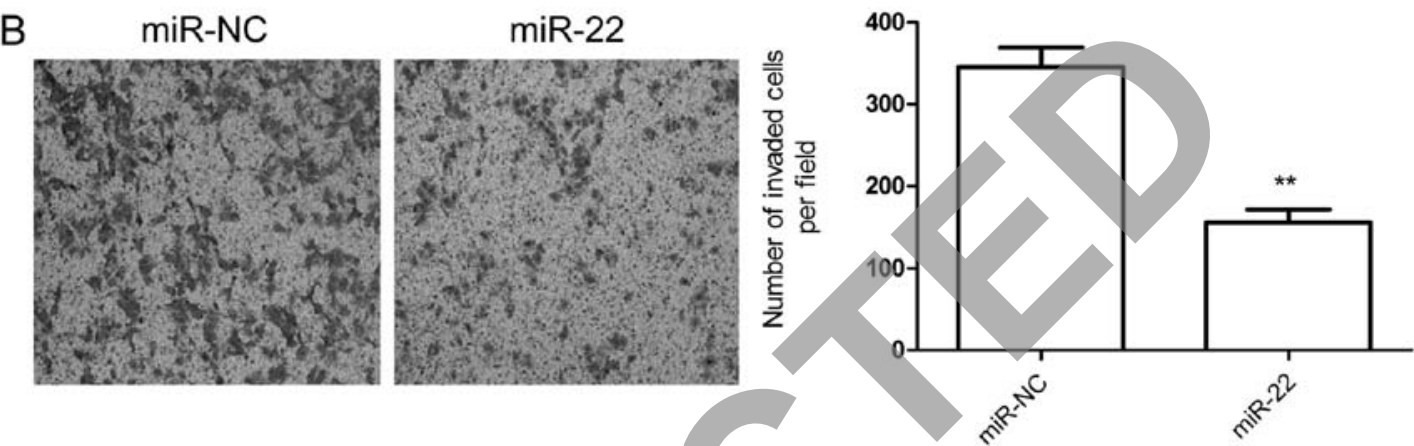

C
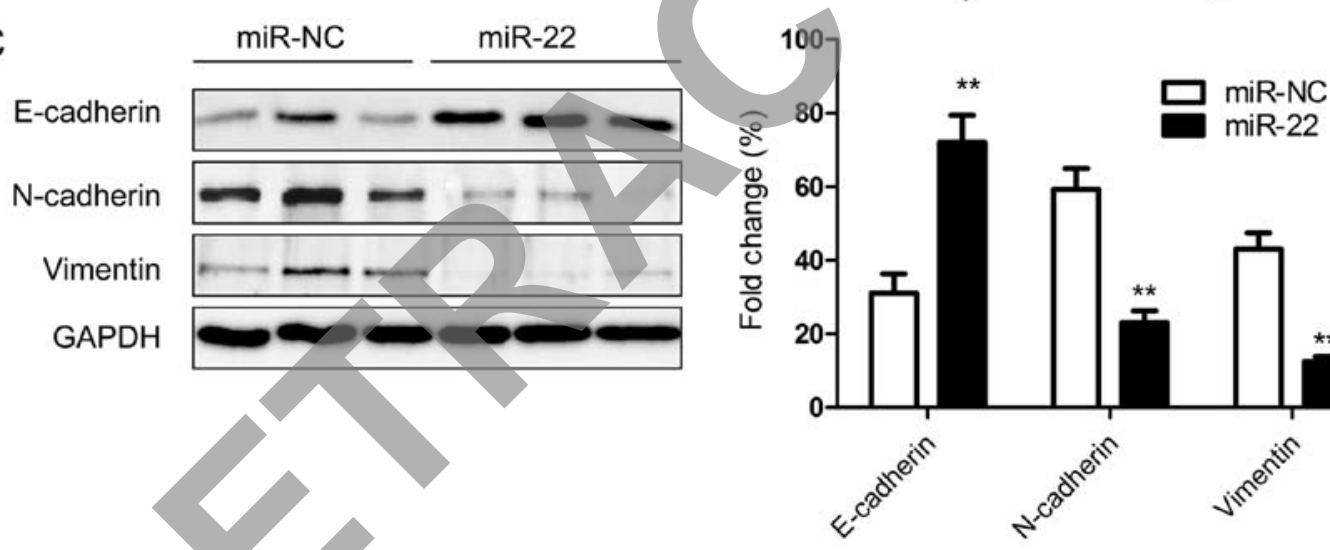

miR-22
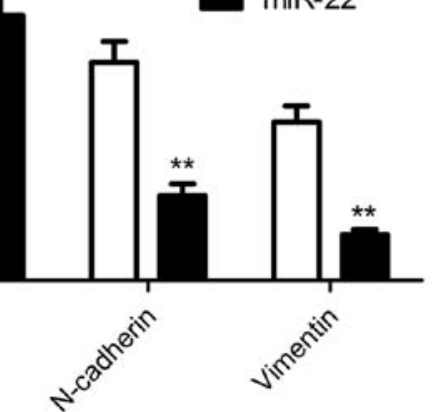

Figure 3. miR-22 inhibits the migration, invasion and epithelial-to-mesenchymal transition (EMT) of renal cancer cells. (A) Cell migration was determined in 786-O cells transfected with miR-22 mimic or miR-NC by wound-healing assay. (B) Cell invasion was determined in 786-O cells transfected with miR-22 mimic or miR-NC by Transwell assay. (C) E-cadherin, N-cadherin and vimentin protein expression levels in 786-O cells transfected with miR-22 mimic or miR-NC were analyzed by western blot analysis. GAPDH was used as a control. The expression levels of E-cadherin, N-cadherin and vimentin were analyzed by densitometry. ${ }^{* *} \mathrm{P}<0.01$ vs. miR-NC group.

cell lines (786-O, ACHN, Caki-1, Caki-2) $(\mathrm{P}<0.05$, Fig. 1A). The 786-O cell line, which possessed the lowest levels of miR-22 expression among the four cell lines, was selected for further study. In addition, we further assessed the expression level of miR-22 in RCC tissues and adjacent normal tissues.

We found that the expression of miR-22 was significantly decreased in RCC tissues as compared with the corresponding adjacent tissues $(\mathrm{P}<0.05$, Fig. 1B).

To investigate the association between miR-22 expression and clinicopathological features in the 50 RCC patients. All RCC samples were divided into miR-22 low-expression group $(\mathrm{n}=24)$ and high-expression group $(\mathrm{n}=26)$, median $(0.467)$ was used as cut-off. We found that miR-22 expression was significantly correlated with histological grade, tumor stage and lymph node metastasis $(\mathrm{P}<0.05)$, but was not significant associated with patient gender, age or tumor size (Table I).
These results indicate that miR-22 is downregulated in renal cancer, suggesting miR-22 may play a key role in the development and progression of renal cancer.

miR-22 suppresses cell proliferation, colony formation, and induces cell arrest and apoptosis in renal cancer cells. To investigate the effect of miR-22 on cell proliferation, colony formation, cell cycle and apoptosis, we performed overexpression experiments in the renal cancer cell line 786-O. Successful overexpression of miR-22 in the cells was confirmed by qRT-PCR (Fig. 2A). MTT assay and colony formation assay showed that overexpression of miR-22 significantly inhibited cell proliferation (Fig. 2B) and colony formation (Fig. 2C). As the proliferation was directly linked to cell cycle distribution, we investigated the effect of miR-22 on RCC cell cycle progression, and found that overexpression 
A

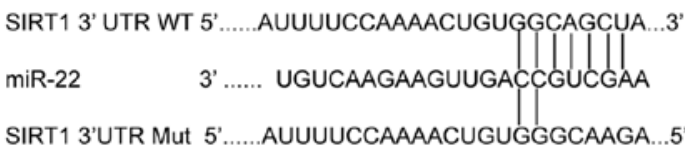

C

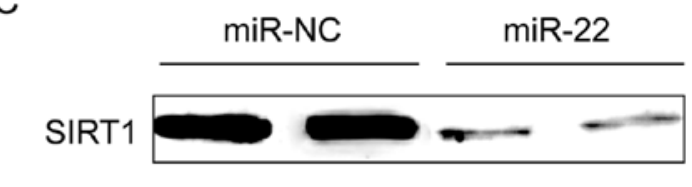

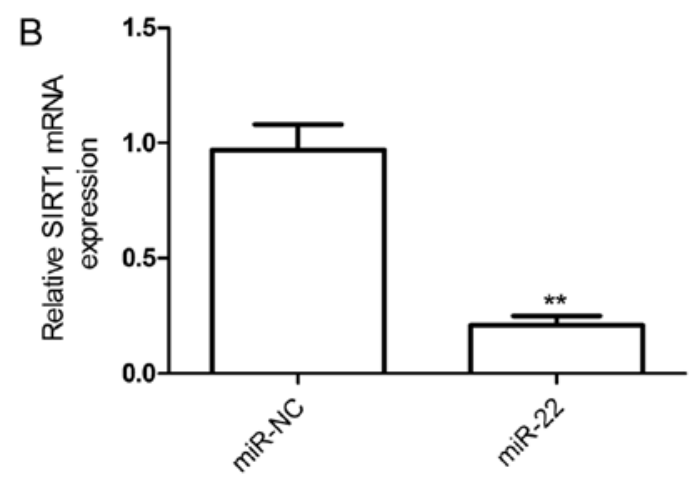

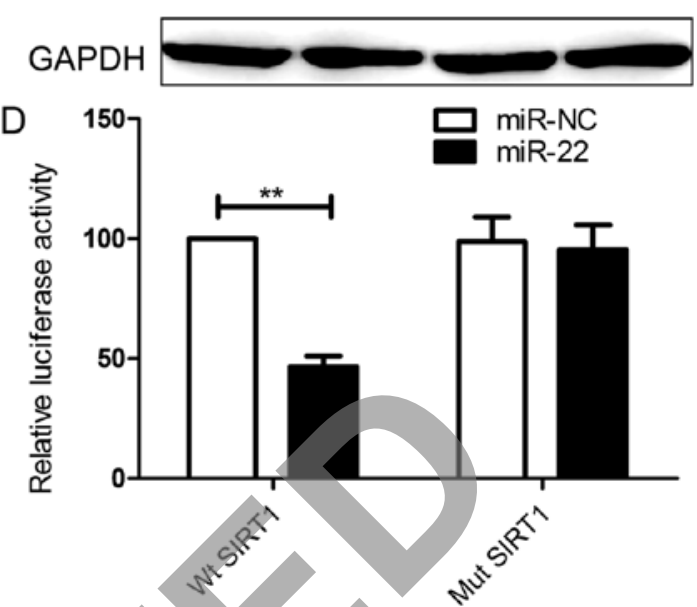

Figure 4. miR-22 targets the 3'UTR of SIRT1 and downregulates its expression. (A) The predicted binding sites for miR-22 in the 3 'UTR of SIRT1 and the mutations in the binding sites are shown. (B) SIRT1 mRNA expression level was determined in 786-O cells transfected with miR-22 mimic or miR-NC by qRT-PCR. GAPDH was used as a control (D). SIRT1 protein expression level was determined in 786-O cells transfected with miR-22 mimic or miR-NC by western blot analysis. GAPDH was used as a control. (D) Luciferase assay in 786-O cells co-transfected with miR-22 or miR-NC and a luciferase reporter plasmid (Wt/Mut 3'UTR IRS1). ${ }^{* *} \mathrm{P}<0.01$ vs. miR-NC group.

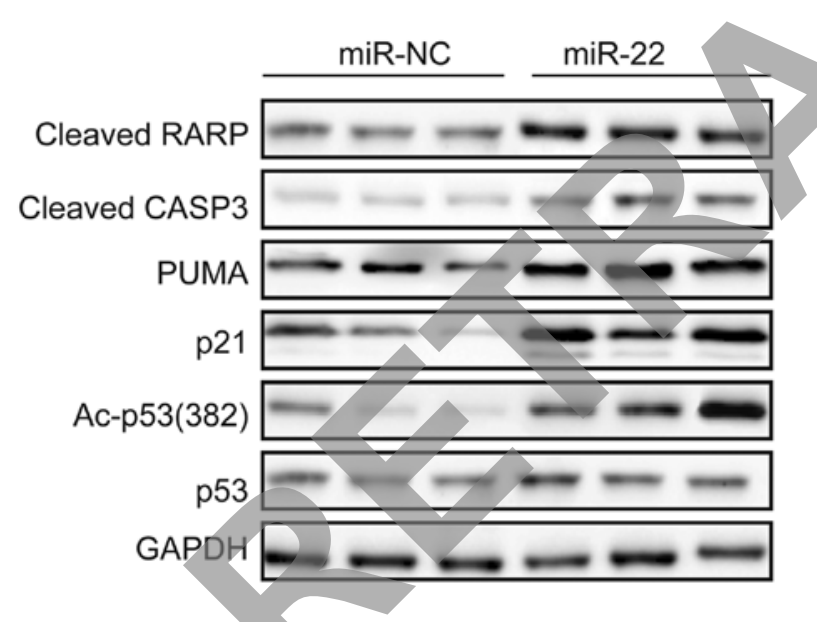

Figure 5. Overexpression of miR-22 activated p53 signal pathway and its downstream protein in RCC cells. p53, Ac-p53(382), p21, PUMA, cleaved CASP 3 and cleaved RARP protein expression in 786-O cells transfected with miR-22 mimic or miR-NC were analyzed by western blot analysis. GAPDH was used as a control.

of miR-22 markedly induced G1 phase arrest and decreased S phase arrest of renal cancer cell lines (Fig. 2D). Cell apoptosis assay showed that overexpression of miR-22 significantly induced apoptosis in RCC cells (Fig. 2E).

miR-22 inhibits the migration, invasion and epithelial-tomesenchymal transition (EMT) of renal cancer cells. The above results showed that miR-22 expression is inversely correlated with the metastatic ability of RCC, therefore, to test whether miR-22 overexpression suppresses tumor cell migration and invasion, the migration and invasion of 786-O cells transfected with miR-22 or miR-NC were determined by wound-healing assay and Transwell assay, respectively. We found that overexpression of miR-22 significantly suppressed migration (Fig. 3A) and invasion (Fig. 3B) in renal cancer cells.

To further investigate whether the inhibitory effect of miR-22 on migration and invasion was mediated by epithelial-mesenchymal transition (ETM) since EMT plays a key role in the invasion of various cancer cells by the transformation of polarized and adherent epithelial cells into motile and invasive mesenchymal cells, we examined the ETM-related to protein expression. As shown in Fig. 3C, overexpression of miR-22 in RCC cells dramatically upregulated E-cadherin protein expression, an epithelial marker, and downregulated $\mathrm{N}$-cadherin and vimentin protein expression, mesenchymal marker, which contribute to suppression of ETM and inhibition of migration and invasion.

miR-22 targets 3'UTR of SIRTI and suppresses the expression of SIRT1. To explore the mechanism of the antitumor effects of miR-22 on RCC cells, bioinformatic database (TargetScan, PicTar, and miRanda) analyses were performed to predict putative miR-22 targets. The results revealed that SIRT1 was a potential target of miR-22 (Fig. 4A). As expected, the qRT-PCR and western blotting results confirmed that miR-22 restoration caused downregulation of SIRT1 expression on mRNA level (Fig. 4B) and protein level (Fig. 4C). To further prove the direct interaction between miR-22 and its targets, we cloned 3'-UTR sequences that contained the predicted target sites (wild type, WT) or mutated sequences (mutant type) of SIRT1 into the psiCHECK-2 vectors. A luciferase reporter assay demonstrated that 786-O cells co-transfected with miR-22 mimic and WT 3'-UTR SIRT1 significantly suppressed the luciferase activity (Fig. 4D), whereas no obvious effect was observed in their negative and mutant controls. These results 
A

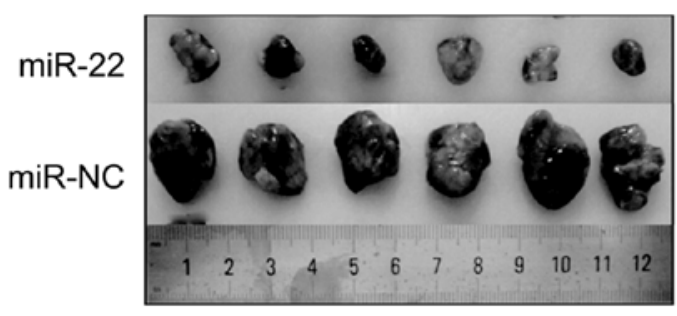

C

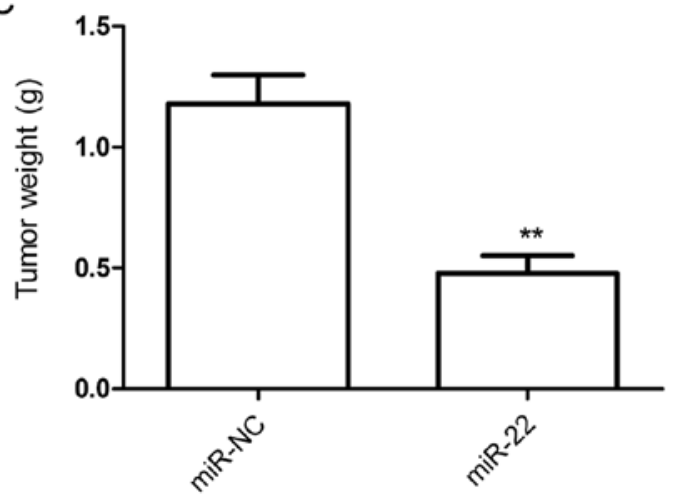

B
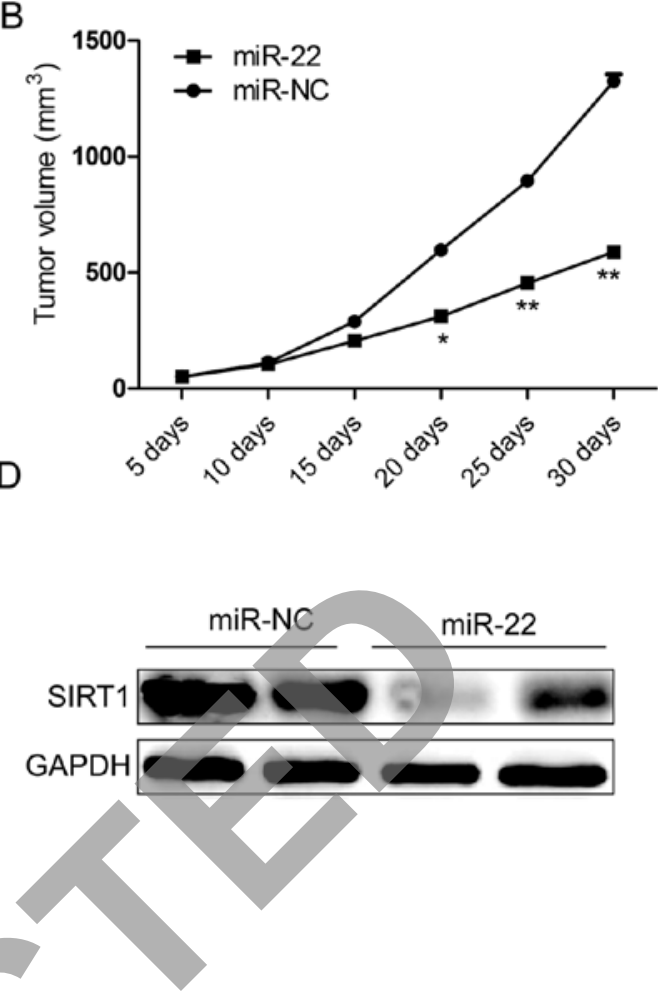

Figure 6. miR-22 suppresses tumor growth in a 768-O xenograft model. (A) Photographs of tumor tissue collected after sacrifice at day 30. (B) Growth curves for tumor volumes in xenografts of nude mice were established based on the tumor volume and measured from day 5 until day 30 . (C) Tumor weights were measured from different groups. (D) SIRT1 protein expression was determined in tumor tissue by qRT-PCR assay. ${ }^{*} \mathrm{P}<0.05,{ }^{* *} \mathrm{P}<0.01 \mathrm{vs}$. miR-NC group.

demonstrated that miR-22 negatively regulates SIRT1 expression by directly binding to the 3'-UTR of SIRT1.

miR-22 activates the p53 signal pathway of renal cancer cells. It has been reported that increased expression of SIRT1 in normal or cancer cells decreases the ability of $\mathrm{p} 53$ by deacetylating p53, leading to decreasing cellular apoptosis $(19,20)$. Therefore, we examined the effect of miR-22 on acetylation of p53. Transfecting miR-22 mimic into 786-O cells decreases SIRT1 (Fig. 4B and C) and increases acetylated p53 (Fig. 5). In addition, we detected p21 and PUMA, both transcriptional targets of p53, and the apoptosis markers cleaved CASP3 and PARP expression in 786-O cells transfected with miR-22 mimic or miR-NC. The results showed that overexpression of miR-22 in 786-O cells increased expression of p21, PUMA, cleaved CASP3 and PARP (Fig. 5). These results suggest that miR-22 indirectly regulates p53 through SIRT1.

miR-22 inhibits tumor growth of RCC in vivo. Finally, we tested whether ectopic expression of miR-22 could influence the growth of breast tumors in vivo. Stably expressing 786-O cells either with miR-22 or miR-NC were injected subcutaneously into nude mice, and the tumor formation was monitored. Cells transfected with miR-NC showed progressive growth, while cells transfected with miR-22 mimic retarded tumor growth compared to miR-NC group (Fig. 6A and B). Thirty days after injection, the nude mice were sacrificed, and the tumors weighed. Our results showed that in miR-22 overexpressing group the weights were significantly lower than in the miR-NC group (Fig. 6C). In addition, the result of western blot analysis showed that SIRT1 protein expression was decreased in miR-22 group compared to the miR-NC group (Fig. 6D). These findings suggested that miR-22 suppressed renal cancer tumorigenicity in vivo by targeting SIRT1.

\section{Discussion}

miRNAs, as a novel class of small, single-stranded, non-coding RNA regulatory molecules, have been demonstrated to play critical roles as either oncogenes or tumor suppressors in various human cancers including renal cancer (21). For example, miR-1 functions as a tumor suppressor and inhibits RCC progression by targeting CDK4, CDK6, Caprin1 and Slug (22). MicroRNA-429 acts as a tumor suppressor, and inhibits cell proliferation, epithelial-mesenchymal transition, and metastasis by direct targeting of BMI1 and E2F3 in renal cell carcinoma (23). MicroRNA-21 (miR-21) functions as an oncogene, and downregulates tumor suppressor PDCD4 and promotes cell transformation, proliferation, and metastasis in renal cell carcinoma (24). The present study first showed that miR-22 could suppress RCC tumor growth in vitro and in vivo by targeting SIRT1, suggesting that miR-22 functions as a tumor suppressor in renal cell carcinoma.

miR-22, located at a fragile cancer-relevant genomic region in chromosome $17(17 \mathrm{p} 13.3)$, is mapped to an exon of the C17orf91 gene (25). microRNA-22 (miR-22) has been shown to be upregulated in prostate cancer (26), and downregulated in breast cancer (12), osteosarcoma (13), esophageal squamous cell carcinoma (14), gastric cancer (15) and colorectal cancer (16). Recent studies have shown that 
miR-22 is involved in various cellular processes related to carcinogenesis, including cell growth, apoptosis, motility, and the cell cycle (17). For example, Xiong et al (27) reported that miR-22 was also downregulated in breast cancer, and it suppressed breast cancer development through directly targeting estrogen receptor $\alpha(\mathrm{ER} \alpha)$ and downstream signaling (12). Guo et al reported that miR-22 inhibits osteosarcoma cell proliferation and migration by targeting HMGB1 and inhibiting HMGB1-mediated autophagy (13). Yang et al demonstrated that miR-22 expression is decreased in human ESCC tissues and cell lines compared with matching adjacent normal tissues and cell lines and restoration of miR-22 in Eca109 and Kyse410 cells significantly inhibited cellular migration and invasion (14). However, miR-22 expression was found to be upregulated in prostate cancer, and its upregulation increased phosphatidylinositol 3-kinase-Akt (PI3K/AKT) signal pathway activation (28). These controversial results of miR-22 in cancer development suggested miR-22 play different roles in different types of cancers. Here, we demonstrated that miR-22 expression is decreased in human RCC tissues and cell lines compared with matching adjacent normal tissues and cell lines, which is consistent with findings from a previous study (18). Restoration of miR-22 in 786-O cells inhibited proliferation, migration and invasion, and induced cell apoptosis in vitro, and suppressed tumor growth in vivo by targeting SIRT1. These results suggest that miR-22 as a tumor suppressor plays a role in the metastasis and progression of RCC.

SIRT1, a class III histone deacetylase, is highly conserved from bacteria to humans and is homologous to silent information regulator 2 (Sir2) in mammals (28). SIRT1 involvement has been shown in regulating various physiological processes, including gene transcription, energy metabolism, cell senescence, glucose metabolism, lipid metabolism, and insulin secretion (29). SIRT1 overexpression is found in prostate (30), ovarian (31), renal (32) colorectal (33) and liver cancers (34), suggesting that SIRT1 may play a role in tumorigenesis. It has been demonstrated that SIRT1 plays an important role in the longevity and cellular senescence of most organisms through directly modulating the p53 signal pathway (35). In addition, recent studies have suggested that SIRT1 expression in cancer cells is regulated by miR-34a (34), miR-126 (36), miR-217 (37), miR-449 (38) and miR-494 (39), and miRNAs regulating SIRT1 are involved in many cellular pathways, including cancer cell proliferation, cycle distribution, migration and invasion (35-39). Here, we confirmed that SIRT1 is a target of miR-22 by luciferase assay, and that upregulation of miR-22 decreased the expression of SIRT1 on mRNA and protein levels. Overexpression of miR-22 actived the p53 signal pathway and its downstream protein. These finding may suggest that miR-22 inhibited RCC growth and metastasis by targeting SIRT1.

In summary, the results presented here demonstrate that miR-22 expression level was downregulated in RCC tissue and cell lines, and its expression level was significantly associated with histological grade, tumor stage and lymph node metastasis. miR-22 functions as a tumor suppressor and suppresses RCC tumor growth in vitro and in vivo by targeting SIRT1. Moreover, miR-22 activated the p53 signal pathway and its downstream proteins, and was able to inhibit
ETM contributing to inhibition of tumor growth and metastasis. These results suggested that miR-22 may be a novel tumor suppressor that blocks the growth of RCC through p53 signaling pathways by targeting SIRT1. Therefore, miR-22 may be a therapeutic target for the treatment of RCC.

\section{References}

1. Siegel R, Ma J, Zou Z and Jemal A: Cancer statistics, 2014. CA Cancer J Clin 64: 9-29, 2014.

2. DeSantis CE, Lin CC, Mariotto AB, Siegel RL, Stein KD, Kramer JL, Alteri R, Robbins AS and Jemal A: Cancer treatment and survivorship statistics, 2014. CA Cancer J Clin 64: 252-271, 2014.

3. Chow WH, Dong LM and Devesa SS: Epidemiology and risk factors for kidney cancer. Nat Rev Urol 7: 245-257, 2010.

4. Pantuck AJ, Zisman A and Belldegrun AS: The changing natural history of renal cell carcinoma. J Urol 166: 1611-1623, 2001.

5. DiBiase SJ, Valicenti RK, Schultz D, Xie Y, Gomella LG and Corn BW: Palliative irradiation for focally symptomatic metastatic renal cell carcinoma: Support for dose escalation based on a biological model. J Urol 158: 746-749, 1997.

6. Fabian MR, Sonenberg N and Filipowicz W: Regulation of mRNA translation and stability by microRNAs. Annu Rev Biochem 79: 351-379, 2010.

7. Guo H, Ingolia NT, Weissman JS and Bartel DP: Mammalian mícroRNAs predominantly act to decrease target mRNA levels. Nature 466: 835-840, 2010.

8. Bartel DP: MicroRNAs: Genomics, biogenesis, mechanism, and function. Cell 116: 281-297, 2004.

9. McManus MT: MicroRNAs and cancer. Semin Cancer Biol 13: 253-258, 2003.

10. Farazi TA, Spitzer JI, Morozov P and Tuschl T: miRNAs in human cancer. J Pathol 223: 102-115, 2011.

1. Garzon R and Marcucci G: Potential of microRNAs for cancer diagnostics, prognostication and therapy. Curr Opin Oncol 24: 655-659, 2012.

12. Kong LM, Liao CG, Zhang Y, Xu J, Li Y, Huang W, Zhang Y, Bian $\mathrm{H}$ and Chen $\mathrm{ZN}$ : A regulatory loop involving miR-22, Sp1, and c-Myc modulates CD147 expression in breast cancer invasion and metastasis. Cancer Res 74: 3764-3778, 2014.

13. Guo S, Bai R, Liu W, Zhao A, Zhao Z, Wang Y, Wang Y, Zhao W and Wang W: miR-22 inhibits osteosarcoma cell proliferation and migration by targeting HMGB1 and inhibiting HMGB1-mediated autophagy. Tumour Biol 35: 7025-7034, 2014.

14. Yang C, Ning S, Li Z, Qin X and Xu W: miR-22 is down-regulated in esophageal squamous cell carcinoma and inhibits cell migration and invasion. Cancer Cell Int 14: 138, 2014.

15. Wang X, Yu H, Lu X, Zhang P, Wang M and Hu Y: miR-22 suppresses the proliferation and invasion of gastric cancer cells by inhibiting CD151. Biochem Biophys Res Commun 445: $175-179,2014$.

16. Zhang G, Xia S, Tian H, Liu Z and Zhou T: Clinical significance of miR-22 expression in patients with colorectal cancer. Med Oncol 29: 3108-3112, 2012.

17. Song SJ and Pandolfi PP: miR-22 in tumorigenesis. Cell Cycle 13: $11-12,2014$.

18. He H, Wang L, Zhou W, Zhang Z, Wang L, Xu S, Wang D, Dong J, Tang $\mathrm{C}$, Tang $\mathrm{H}$, et al: MicroRNA expression profiling in clear cell renal cell carcinoma: Identification and functional validation of key miRNAs. PLoS One 10: e0125672, 2015.

19. Vaziri H, Dessain SK, Ng Eaton E, Imai SI, Frye RA, Pandita TK, Guarente L and Weinberg RA: hSIR2(SIRT1) functions as an NAD-dependent p53 deacetylase. Cell 107: 149-159, 2001.

20. Langley E, Pearson M,Faretta M,Bauer UM, Frye RA, Minucci S, Pelicci PG and Kouzarides T: Human SIR2 deacetylates p53 and antagonizes PML/p53-induced cellular senescence. EMBO J 21: 2383-2396, 2002.

21. Li JY, Yong TY, Michael MZ and Gleadle JM: Review: The role of microRNAs in kidney disease. Nephrology (Carlton) 15: $599-608,2010$.

22. Xiao H, Zeng J, Li H, Chen K, Yu G, Hu J, Tang K, Zhou H, Huang Q, Li A, et al: miR-1 downregulation correlates with poor survival in clear cell renal cell carcinoma where it interferes with cell cycle regulation and metastasis. Oncotarget 6: 13201-13215, 2015. 
23. Qiu M,Liang Z, Chen L, Tan G, Wang K, Liu L, Liu J and Chen H: MicroRNA-429 suppresses cell proliferation, epithelial-mesenchymal transition, and metastasis by direct targeting of BMI1 and E2F3 in renal cell carcinoma. Urol Oncol 33: 332.e9-e18, 2015.

24. Li X, Xin S, He Z, Che X, Wang J, Xiao X, Chen J and Song X: MicroRNA-21 (miR-21) post-transcriptionally downregulates tumor suppressor PDCD4 and promotes cell transformation, proliferation, and metastasis in renal cell carcinoma. Cell Physiol Biochem 33: 1631-1642, 2014.

25. Gurha P, Abreu-Goodger C, Wang T, Ramirez MO, Drumond AL, van Dongen S, Chen Y, Bartonicek N, Enright AJ, Lee B, et al: Targeted deletion of microRNA-22 promotes stress-induced cardiac dilation and contractile dysfunction. Circulation 125 2751-2761, 2012.

26. Pasqualini L, Bu H, Puhr M, Narisu N, Rainer J, Schlick B, Schäfer G, Angelova M, Trajanoski Z, Börno ST, et al: miR-22 and miR-29a are members of the androgen receptor cistrome modulating LAMC1 and Mcl-1 in prostate cancer. Mol Endocrinol 29: 1037-1054, 2015.

27. Xiong J1, Yu D, Wei N, Fu H, Cai T, Huang Y, Wu C, Zheng X, Du Q, Lin D, Liang Z: An estrogen receptor alpha suppressor, microRNA-22, is downregulated in estrogen receptor alpha-positive human breast cancer cell lines and clinical samples. FEBS J 277: 1684-1694.

28. Poliseno L, Salmena L, Riccardi L, Fornari A, Song MS, Hobbs RM, Sportoletti P, Varmeh S, Egia A, Fedele G, et al: Identification of the miR-106b 25 microRNA cluster as a proto-oncogenic PTEN-targeting intron that cooperates with its host gene MCM7 in transformation. Sci Signal 3: ra29, 2010.

29. Dong YJ, Liu N, Xiao Z, Sun T, Wu SH, Sun WX, Xu ZG and Yuan H: Renal protective effect of sirtuin 1. J Diabetes Res 2014: 843786, 2014.

30. Huffman DM, Grizzle WE, Bamman MM, Kim JS, Eltoum IA, Elgavish A and Nagy TR: SIRT1 is significantly elevated in mouse and human prostate cancer. Cancer Res 67: 6612-6618 2007.

31. Jang KY, Kim KS, Hwang SH, Kwon KS, Kim KR, Park HS Park BH, Chung MJ, Kang MJ, Lee DG, et al: Expression and prognostic significance of SIRT1 in ovarian epithelial tumours. Pathology 41: 366-371, 2009.
32. Jung YJ, Lee JE, Lee AS, Kang KP, Lee S, Park SK, Lee SY, Han MK, Kim DH and Kim W: SIRT1 overexpression decreases cisplatin-induced acetylation of $\mathrm{NF}-\kappa \mathrm{B}$ p65 subunit and cytotoxicity in renal proximal tubule cells. Biochem Biophys Res Commun 419: 206-210, 2012.

33. Stünkel W, Peh BK, Tan YC, Nayagam VM, Wang X, Salto-Tellez M, Ni B, Entzeroth M and Wood J: Function of the SIRT1 protein deacetylase in cancer. Biotechnol J 2: 1360-1368, 2007.

34. Chen J, Zhang B, Wong N, Lo AW, To KF, Chan AW, Ng MH, Ho CY, Cheng SH, Lai PB, et al: Sirtuin 1 is upregulated in a subset of hepatocellular carcinomas where it is essential for telomere maintenance and tumor cell growth. Cancer Res 71 : 4138-4149, 2011

35. Yamakuchi M, Ferlito M and Lowenstein CJ: miR-34a repression of SIRT1 regulates apoptosis. Proc Natl Acad Sci USA 105: 13421-13426, 2008.

36. Xu JQ, Liu P, Si MJ and Ding XY: MicroRNA-126 inhibits osteosarcoma cells proliferation by targeting Sirt1. Tumour Biol 34: 3871-3877, 2013

37. Deng S, Zhu S, Wang B, Li X, Liu Y, Qin Q, Gong Q, Niu Y, Xiang C, Chen J, et al: Chronic pancreatitis and pancreatic cancer demonstrate active epithelial-mesenchymal transition profile, regulated by miR-217-SIRT1 pathway. Cancer Lett 355: 184-191, 2014.

38. Zhang H, Feng Z, Huang R, Xia Z, Xiang G and Zhang J: MicroRNA-449 suppresses proliferation of hepatoma cell lines through blockade lipid metabolic pathway related to SIRT1. Int J Oncol 45: 2143-2152, 2014

39. Liu Y, Li X, Zhu S, Zhang JG, Yang M, Qin Q, Deng SC, Wang B, Tian K, Liu L, et al: Ectopic expression of miR-494 inhibited the proliferation, invasion and chemoresistance of pancreatic cancer by regulating SIRT1 and c-Myc. Gene Ther: Apr 28, 2015 (Epub ahead of print). doi: 10.1038/gt.2015.39. 